\title{
Transmission Through a Quantum Dynamical Delta Barrier
}

\author{
T. Brandes and J. Robinson \\ Dept. of Physics, UMIST, PO Box 88, Manchester M60 1QD, UK
}

\begin{abstract}
We discuss electron scattering in a one-dimensional delta barrier potential with either time-dependent coupling constant (classical model) or a coupling constant that is linear in a boson coordinate (quantum model). We find an exact continued fraction solution and Fano like resonances in the transmission coefficient. In the quantum model, energies for perfect transmission exist below the first sideband threshold.
\end{abstract}

\section{Introduction and Model}

Simple models for the interaction between fermions and bosons continue to be fascinating, as often very non-trivial results can be obtained from even the most primitive Hamiltonians. Exactly solvable models for the interaction of photons or phonons with electrons in quantum dots [1] or quasi-one-dimensional systems [2, 3] provide the best examples, as they often provide a deep insight into rich and complex physics.

In this contribution, we re-consider a simple model for a single electron of mass $m$ in one dimension that interacts with a delta-barrier through a coupling parameter that itself is a dynamical quantity. The Hamiltonian is written as

$$
H=\frac{p^{2}}{2 m}+\delta(x)\left\{g_{0}+g_{1}\left[a^{\dagger}+a\right]\right\}+\Omega a^{\dagger} a .
$$

Here, $a^{\dagger}$ creates a boson of frequency $\Omega$ and $g_{1}\left[a^{\dagger}+a\right]$ is a dynamical contribution on top of the static coupling constant $g_{0}$. The constant zero point energy is omitted since it merely shifts the energy scale by $\Omega / 2$. The lattice version of this model was originally introduced by Gelfand, Schmitt-Rink and Levi [2] years ago in the study of tunneling in presence of phonons, and was shortly afterwards compared to a corresponding time-dependent classical Hamiltonian [ $₫$ ], the continuous version of which reads

$$
H_{\mathrm{cl}}(t)=\frac{p^{2}}{2 m}+\delta(x)\left\{g_{0}+2 g_{1} \cos (\Omega t)\right\} .
$$

$H_{\mathrm{cl}}(t)$ is obtained as the interaction picture Hamiltonian of Eq.(11) with respect to $H_{B}=\Omega a^{\dagger} a$, after replacing the boson operators by $a^{\dagger}=a=1$.

In its time-dependent version, Eq.(2) has subsequently been used as a model for scattering in quasi-one-dimensional quantum wires by Bagwell and co-workers [3, 5 ], who found Fano-type resonances in the transmission coefficient as a function of the energy of an incident electron. It soon turned out that the scattering properties of this Hamiltonian are quite intriguing as they very much depend on the relative sign and strength of the two coupling parameters $g_{0}$ and $g_{1}$. The interplay between evanescent modes, quasibound states [5], and the behaviour of the transmission amplitude in the complex energy plane [6, 7] have been studied recently.

Our focus here is on the quantum version Eq. (1) of the model and its peculiarities in comparison with $H_{\mathrm{cl}}$. It turns out that beside transmission zeroes, there are points of perfect 
transparency in the Fano resonance that only appear in the model $H$ but not in $H_{\mathrm{cl}}$. Perfect transmission and Fano resonances have been found recently in the transmission of phonons through non-linear chains without delta impurities [8, 9]. Although not discussed in detail here, these results indicate that there still is rich and non-trivial behavior to be discovered from models like Eq.(11).

\section{Transmission Coefficient}

The total wave function $|\Psi\rangle$ of the coupled electron-boson system can be expanded in the oscillator basis $\{|n\rangle\}$ as

$$
\langle x \mid \Psi\rangle=\sum_{n=0}^{\infty} \psi_{n}(x)|n\rangle
$$

with wave function coefficients $\psi_{n}(x)$ depending on the position $x$ of the electron. We solve the stationary Schrödinger equation at total energy $E>0$, implying a scattering condition for the electron part of the wave function in demanding that there is no electron incident from the right. For $x \neq 0$, the $\psi_{n}(x)$ are superpositions of plane waves if $E$ is above the threshold for the $n-t h$ boson energy,

$$
\begin{aligned}
& \psi_{n}(x<0)=a_{n} e^{i k_{n} x}+b_{n} e^{-i k_{n} x} \\
& \psi_{n}(x>0)=t_{n} e^{i k_{n} x}, \quad k_{n} \equiv \sqrt{E-n \Omega}, \quad E>n \Omega,
\end{aligned}
$$

whereas normalizabale evanescent modes occur if $E$ is below the threshold,

$$
\begin{aligned}
& \psi_{n}(x<0)=b_{n} e^{\kappa_{n} x} \\
& \psi_{n}(x>0)=t_{n} e^{-\kappa_{n} x}, \quad \kappa_{n} \equiv \sqrt{n \Omega-E}, \quad E<n \Omega .
\end{aligned}
$$

Here and in the following we set $\hbar=2 m=1$. We impose the condition that the boson is in its ground state for an electron incoming from the left,

$$
a_{n}=\delta_{n, 0},
$$

where we set the corresponding amplitude $A=A_{0}$ to unity. Continuity of $\psi_{n}(x)$ at $x=0$ yields $a_{n}+b_{n}=t_{n}$ for all $n$, whereas the jump in derivative of $\psi_{n}(x)$ across the delta barrier leads to a recursion relation for the transmission amplitudes $t_{n}$,

$$
g_{1} \sqrt{n} t_{n-1}+\left(g_{0}-2 i \gamma_{n}\right) t_{n}+g_{1} \sqrt{n+1} t_{n+1}=-2 i \gamma_{n} \delta_{n, 0}
$$

where the $\gamma_{n}$ are real (imaginary) above (below) the boson energy $n \Omega$,

$$
\gamma_{n}=k_{n} \theta(E-n \Omega)+i \kappa_{n} \theta(n \Omega-E) .
$$

The total transmission coefficient $T(E)$ is obtained from the sum over all propagating modes,

$$
T(E)=\sum_{n=0}^{[E / \Omega]} \frac{k_{n}(E)}{k_{0}(E)}\left|t_{n}(E)\right|^{2},
$$

where the sum runs up to the largest $n$ such that $k_{n}$ remains real.

\section{Matrix Representation and Continued Fractions}

Although Eq.(9) is a finite sum, its evaluation requires the solution of the infinite recursion relation Eq.(7) due to the fact that the propagating modes are coupled to all evanescent modes. 
The transmission amplitudes can be determined from the linear equation

$$
\begin{aligned}
& M \mathbf{t}=\mathbf{a}, \quad \mathbf{t}=\left(t_{0}, t_{1}, t_{2}, \ldots\right), \quad \mathbf{a}=\left(-2 i \gamma_{0}, 0,0, \ldots\right) \\
& M=\left(\begin{array}{cccc}
g_{0}-2 i \gamma_{0} & \sqrt{1} g_{1} & 0 & \\
\sqrt{1} g_{1} & g_{0}-2 i \gamma_{1} & \sqrt{2} g_{1} & 0 \\
0 & \sqrt{2} g_{1} & g_{0}-2 i \gamma_{2} & \ddots \\
& 0 & \ddots & \ddots
\end{array}\right) .
\end{aligned}
$$

Numercally, this can easily been solved by truncation of the matrix $M$. Alternatively, one can solve Eq.(10) recursively which actually is numerically more efficient. For example, the result for the zero-channel transmission amplitude $t_{0}(E)$ can be written in a very intuitive form: defining the 'Greens function' $G_{0}(E)$ by

$$
G_{0}(E) \equiv\left[-2 i \gamma_{0}(E)+g_{0}\right]^{-1},
$$

one can write $t_{0}(E)$ with the help of a recursively defined 'self energy' $\Sigma^{(N)}(E)$,

$$
t_{0}(E)=\frac{-2 i \gamma_{0}(E)}{G_{0}^{-1}(E)-\Sigma^{(1)}(E)}, \quad \Sigma^{(N)}(E)=\frac{N g_{1}^{2}}{G_{0}^{-1}(E-N \Omega)-\Sigma^{(N+1)}(E)}(12)
$$

In fact, using $\gamma_{n}(E)=\gamma_{0}(E-n \Omega)$, the self energy $\Sigma^{(1)}(E)$ can be represented as a continued fraction

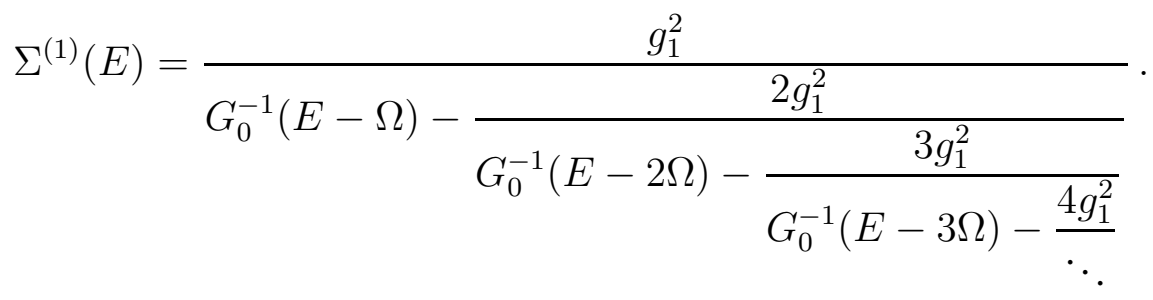

This demonstrates that $t_{0}(E)$ depends on $g_{1}$ only through $g_{1}^{2}$.

Truncating the matrix $M$ to a $N \times N$ matrix corresponds to the approximation that sets $\Sigma^{(N)}(E) \equiv 0$ and recursively solves Eq. (12) for $\Sigma^{(N-1)}(E)$ down to $\Sigma^{(1)}(E)$. For example, truncating at $N=2$ we obtain the approximation

$$
t_{0, N=2}(E)=\frac{-2 i \gamma_{0}(E)}{G_{0}^{-1}(E)-\Sigma_{N=2}^{(1)}(E)}=\frac{-2 i \gamma_{0}(E)}{-2 i \gamma_{0}(E)+g_{0}-\frac{g_{1}^{2}}{-2 i \gamma_{1}(E)+g_{0}}} .
$$

An important observation can be made with respect to the stability of our theory for large coupling constants $g_{1}$. In fact, the truncation at $N+1$ is only consistent if the truncated self energy $\Sigma^{(N)}(E)$ is a small correction to the inverse 'free propagator',

$$
N g_{1}^{2} /\left|G_{0}^{-1}(E-N \Omega)\right|<\left|G_{0}^{-1}(E-(N-1) \Omega)\right|,
$$

which by use of Eq. (11) at large $N$ implies $N g_{1}^{2}<4 N \Omega$ or $g_{1}<2 \sqrt{\Omega}$. The tridiagonal form of the matrix, Eq. (10), actually implies that the method based on the recursion Eq. (7) is perturbative in the coupling $g_{1}$ to the boson. We conjecture that for $g_{1}$ above the critical value, the perturbation based on the oscillator basis $\{|n\rangle\}$ used here breaks down. A similar breakdown of numerical approaches that start from a weak coupling regime in single boson Hamiltonians is known from the Rabi Hamiltonian [10], i.e. the coupling of a single boson mode to a spin $1 / 2$.

We mention that the lattice version of the present model would be a natural starting point for a more detailed analysis of the strong coupling (small polaron) limit. 

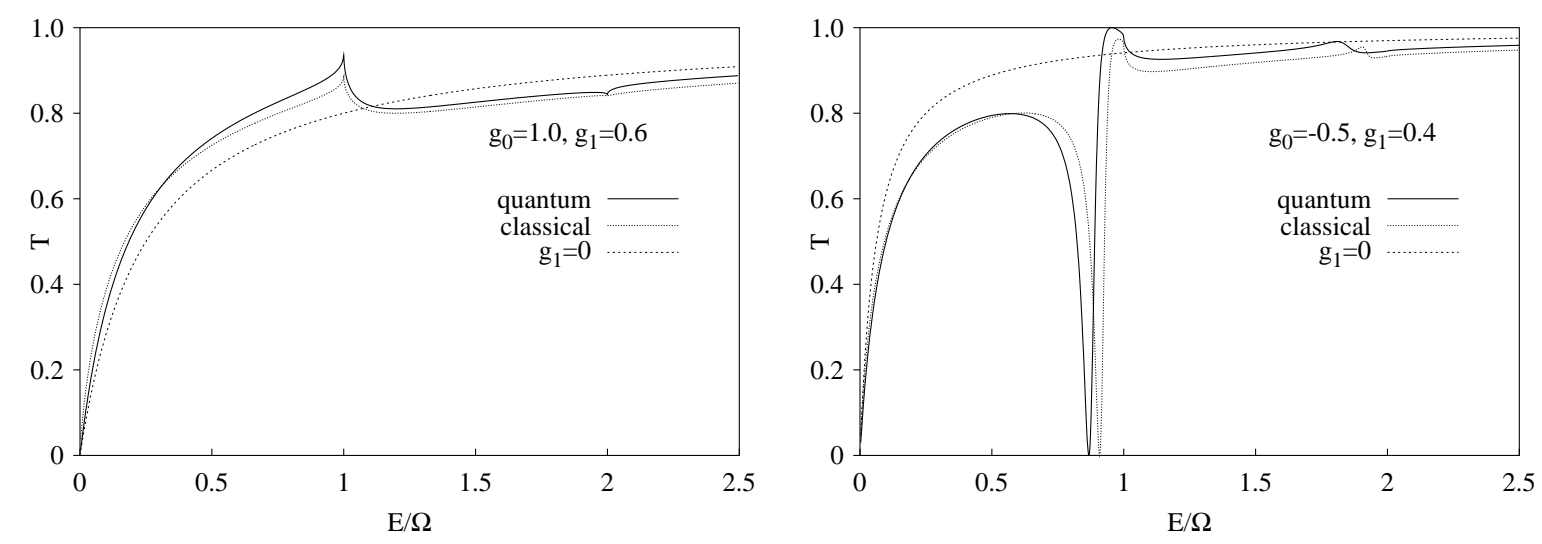

Figure 1. Transmission coefficient through a dynamical one-dimensional delta barrier with repulsive $\left(g_{0}>0\right.$, left $)$ and attractive $\left(g_{0}<0\right.$, right) static part, cf. Eq. (11) and (2). $E$ is the energy of the incident particle.

\section{Comparison to the Classical Case}

The recursion relation corresponding to Eq. (7) for the classical time-dependent Hamiltonian, Eq. (2), was derived and discussed by Bagwell and Lake [5],

$$
g_{1} t_{n-1}+\left(g_{0}-2 i \gamma_{n}\right) t_{n}+g_{1} t_{n+1}=-2 i \gamma_{n} \delta_{n, 0}, \quad n=0, \pm 1, \pm 2, \ldots
$$

Here, $t_{n}$ is the coefficient of the time-dependent electron wave function in photon side-band $n$, where $n$ runs through positive and negative integers $n$. In further contrast to the recursion relation Eq. (7), there are no factors $\sqrt{n}$ and $\sqrt{n+1}$ multiplying the coupling constant $g_{1}$. This latter fact is an important difference to the quantum case where these terms lead to the factors $N$ that multiply $g_{1}^{2}$ in the self energies $\Sigma^{(N)}(E)$, Eq. (12). This difference is eventually responsible for the breakdown of the perturbative approach for large $g_{1}$ in the quantum case.

A continued fraction representation of $t_{0}(E)$ for the classical case has been derived recently by Martinez and Reichl [6]. The corresponding matrix defining the transmission amplitudes $\mathbf{t}_{\mathrm{cl}}=\left(\ldots, t_{-2}, t_{-1}, t_{0}, t_{1}, t_{2}, \ldots\right)$ in the classical case is the infinite tridiagonal matrix $M_{\mathrm{cl}}$ with $g_{0}-i \gamma_{n}$ on the diagonal and $g_{1}$ on the lower and upper diagonals,

$$
M_{\mathrm{cl}}=\left(\begin{array}{ccccc}
\ddots & \ddots & 0 & & \\
\ddots & g_{0}-2 i \gamma_{-1} & g_{1} & 0 & \\
0 & g_{1} & g_{0}-2 i \gamma_{0} & g_{1} & 0 \\
& 0 & g_{1} & g_{0}-2 i \gamma_{1} & \ddots \\
& & 0 & \ddots & \ddots
\end{array}\right) .
$$

Fig. (11) shows a comparison between the transmission coefficient $T(E)$, Eq. (9), for the quantum and the classical barrier. In the repulsive case with $0<g_{1}<g_{0}$, the dynamical part of the barrier is essentially a weak perturbation to the unperturbed $\left(g_{1}=0\right)$ case. Additional structures (cusps) appear at the boson (photo side-band) energies $n \Omega$ although the overall $T(E)$-curve resembles the $\left(g_{1}=0\right)$ case.

The more interesting case occurs for barriers with an attractive static part, $g_{0}<0$ (Fig. (11), right). A Fano type resonance appears below the first threshold $E=\Omega$ where the transmission coefficient has a zero in both the classical and the quantum case. In the classical case, this is a well-known phenomenon [5]: the transmission zero for weak coupling (small 

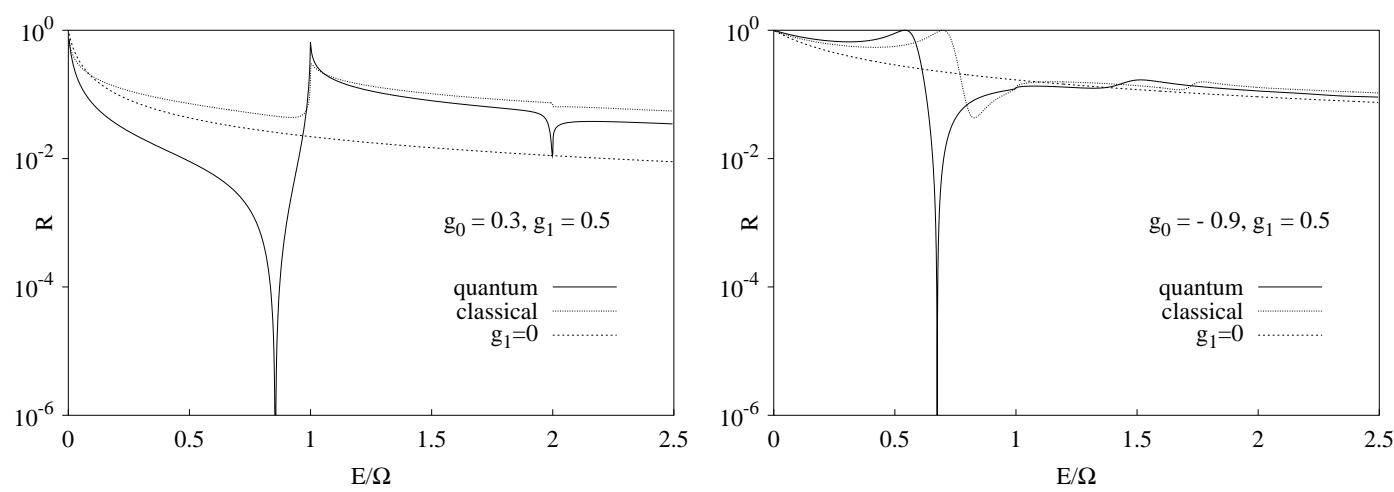

Figure 2. Logarithmic plot of reflection coefficient $R \equiv 1-T$ for dynamical delta barrier with static repulsive $\left(g_{0}>0\right.$, left $)$ and attractive $\left(g_{0}<0\right.$, right) core.

$\left.g_{1}\right)$ shows up when the Fano resonance condition

$$
2 \kappa_{1}(E)+g_{0}=0
$$

is fulfilled.There, the energy of the electron in the first side channel $(n=1)$ coincides with the bound state of the attractive delta barrier potential, $E-\Omega=-g_{0}^{2} / 4$. In the quantum case, the self energy in Eq.(12) diverges at the zeros of $T(E)$,

$$
\left[\Sigma^{(1)}(E)\right]^{-1}=0 .
$$

For $g_{1} \rightarrow 0, \Sigma^{(1)}(E) \rightarrow \Sigma_{N=2}^{(1)}(E)=g_{1}^{2} /\left(2 \kappa_{1}(E)+g_{0}\right)$, cf. Eq.(14), and the two conditions Eq.(18) and Eq.(19) coincide.

\section{Perfect Transparency}

The most interesting feature in the scattering properties of the dynamical quantum barrier is the appearance of an energy close to the first channel $(n=1)$ threshold where perfect transmission $T(E)=1$ occurs. This is clearly visible in the vanishing of the reflection coefficient, $R(E) \equiv 1-T(E)$, in the logarithmic plot Fig. (2). For a repulsive static part, $g_{0}=0.3$, this occurs at an energy below the energy where the reflection coefficient comes close to unity, and above that energy if the static part is attractive $\left(g_{0}=-0.9\right)$. In contrast, in the classical case the reflection coefficient never reaches zero in neither the repulsive nor the attractive case. This contrast becomes even more obvious in a two-dimensional plot where the zeros in $R$ correspond to 'ridges' in the $g_{0}-E$ plane, cf. Fig. (3).

Perfect transparency $(R=1-T=0)$ can be understood by considering the transmission amplitude $t_{0}(E)$ which determines the total transmission below the first sideband threshold. Recalling that $t_{0}(E)=-2 i k_{0} /\left(-2 i k_{0}+g_{0}-\Sigma^{(1)}(E)\right)$, in the quantum case the transmission coefficient becomes unity when

$$
g_{0}-\Sigma^{(1)}(E)=0 .
$$

Our exact continued fraction expression for the self energy, Eq.(13), implies that for $0<E<$ $\Omega, \Sigma^{(1)}(E)$ is real because $G_{0}^{-1}(E-n \Omega)=2 \sqrt{n \Omega-E}+g_{0}$ is real for $n \geq 1$. The condition Eq.(20) then means that the self energy exactly renormalizes the static part $g_{0}$ of the scattering potential to zero.

For small $g_{1}$, we can use our perturbative expression corresponding to truncating the matrix $M$, Eq.(10), to a two-by-two matrix. The perfect transparency condition Eq.(20) then 

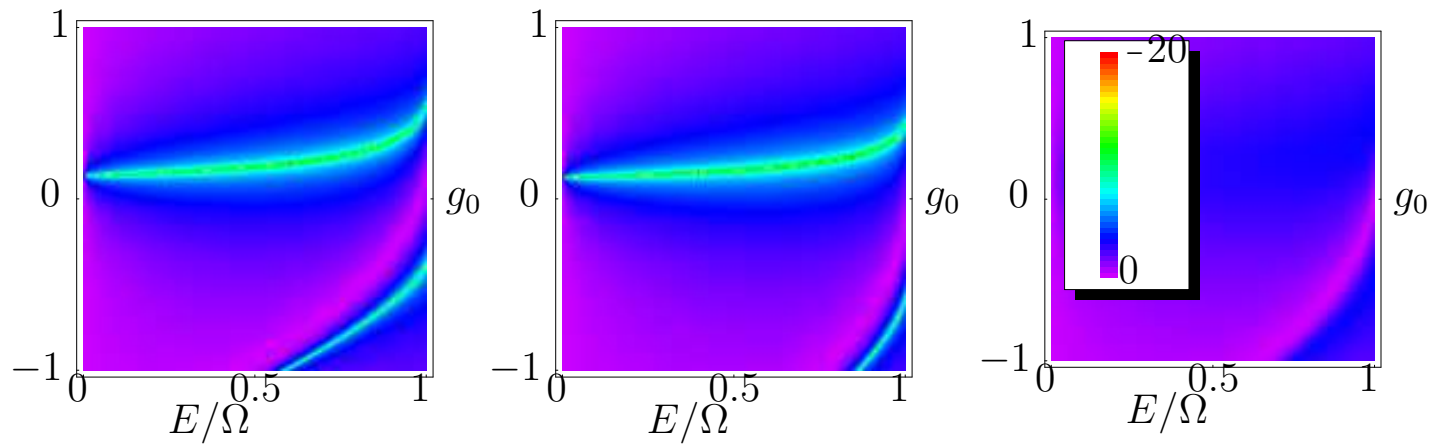

Figure 3. Density plot of $\ln R$ (reflection coefficient) for the quantum delta barrier at $g_{1}=0.5$. Exact solution from Eq. (12) (left), from the $N=2$ truncation Eq. (14) (center), and from the classical model Eq.(2) (right). The light 'ridges' correspond to curves of perfect transmission $T$, cf. Eqs. (21) and Eqs. (22).

becomes

$$
g_{0}-\frac{g_{1}^{2}}{2 \kappa_{1}(E)+g_{0}}=0, \quad 0<E<\Omega, \quad(N=2 \text { truncation. }),
$$

which determines the position of the perfect transmission energy. The solution of Eq.(21) defines two curves in the $E-g_{0}$-plane with perfect transmission for $0<E<\Omega$,

$$
g_{0}=-\sqrt{\Omega-E} \pm \sqrt{\Omega-E+g_{1}^{2}} .
$$

These two curves can be clearly identified in the logarithmic density plots of the reflection coefficient $R=1-T$, cf. Fig. (3). The $N=2$ approximation to the transmission amplitude, Eq.(14), turns out to reproduce these features quite well even at moderate coupling constants $g_{1}$.

\section{Conclusions}

The above analysis of the two models Eq. (11) and Eq. (2) has revealed some interesting differences between scattering properties of simple electron-boson models and their classical counter-part. The strong coupling limit of the quantum model and its extension to more complicated situations like multi-channel scattering remain to be explored.

This work was supported by the UK project EPSRC GR44690/01, and the UK Quantum Circuits Network.

[1] T. Stauber, R. Zimmermann, and H. Castella, Phys. Rev. B 62, 7336 (2000).

[2] B.. Y. Gelfand, S. Schmitt-Rink, and A. F. J. Levi, Phys. Rev. Lett. 62, 1683 (1989).

[3] P. F. Bagwell, Phys. Rev. B 41, 10354 (1990).

[4] J.-M. Lopez-Castillo, C. Tannous, and J.-P. Jay-Gerin, Phys. Rev. A 41, 2273 (1990).

[5] P. F. Bagwell and R. K. Lake, Phys. Rev. B 46, 15329 (1992).

[6] D. F. Martinez and L. E. Reichl, Phys. Rev. B 64, 245315 (2001).

[7] S. W. Kim, H-K. Park, H.-S. Sim, and H. Schomerus, cond-mat/0203391.

[8] S. Kim, C. Baesens, and R. S. MacKay, Phys. Rev. E 56, R4955 (1997).

[9] S. W. Kim and S. Kim, Phys. Rev. B 63, 212301 (2001).

[10] I. I. Rabi, Phys. Rev. 51, 652 (1937); R. F. Bishop and C. Emary, J. Phys. A 34, 5635 (2001). 